\title{
Stem cell research: State of the art
}

\author{
Sandro Eridani ${ }^{1 *} \&$ Lidia Cova ${ }^{2}$ \\ ${ }^{1}$ National Research Council, ITB-CNR, Milan, Italy; ${ }^{2}$ IRCCS Ospedale Maggiore, University of Milan and IRCCS \\ Auxologico Inst., Milan, Italy \\ (*Author for correspondence; E-mail: eridani@itb.cnrit; Fax: +392 26422770)
}

\section{Introduction}

The present issue aims to provide a general evaluation of the state of the art in a field, which is surrounded by a very unusual attention by all quarters of the scientific, social and political arena. The reason why all this is happening is of course related to the outstanding developments in stem cell research, possibly leading to applications in human biology and medicine, which were unthinkable only a few years ago.

We want therefore to present an updated and objective series of reviews of the state of the art in this area: is however important to point out that, in a series of papers on related subjects, a modest degree of overlapping topics and considerations is inevitable. We also have not avoided controversial issues, but of course any opinion is the responsabilty of the scientists actually involved. We hope that this work will help interested people to form their own judgment on such problems.

A very early definition of stem cells was of 'ancestral cells of the germ line' (Wilson 1898). Later, however, it became apparent that stem cells are not confined to the germ line and that they are endowed with specific properties; therefore Laitha proposed in 1970 a classical definition: "cells which have the capacity to divide simmetrically to expand their number and asimmetrically in order to self-renew and give rise to a differentiated progeny'.

A variety of recent reviews have dealt with the general topic of stem cell characterization, differentiation and versatility (Van der Kooy and Weiss, 2000; Snyder and Vescovi, 2000; Weissman, 2000; Eridani, 2001; Blau et al., 2001; Lovell-Badge, 2001).

An updated review of mathematical and experimental models for stem cell renewal and differentiation is presented in this issue by Viswanathan and Zandstra.
A significant, very recent advance has been the recognition that both murine and human stem cells express a higher number of genes than differentiated cells, although their function is often still unknown (Ramalho Santos et al., 2002). Moreover, they share a variety of expressed gene products, defining conserved regulatory pathways; in mice the genetic programme of haemopoietic stem cells is shared with embryonic and neural stem cells. It is suggested that this set of gene products may represent a 'signature' of stem cells (Ivanova et al., 2002).

Among the germ cells, blastocyst cells do not selfrenew and are not present over the entire lifespan of the body; also, oocytes are produced in a limited number shortly after birth. Therefore, according to Van der Kooy and Weiss (2000), such elements cannot be called stem cells: only spermatogonial cells satisfy the requirement for real stem cells.

Embryonic Stem Cells are multipotent stem cells which can be derived from the blastocyst during the early stages of mammalian development. There are two types of embryonic cells: EG (Embryonic Germ), obtained from primordial germ cells taken from the developing gonadal ridges of human fetuses and ES, derived from the inner cell mass of preimplantation embryos. ES can be indefinitely maintained in an undifferentiated state, can colonize all tissues including germline and they can originate, using transgenic and gene targeting technology, animal models for diseases (Smith, 2001).

Human ES express also a wide range of differentiation markers, can proliferate for long time in culture, can be cryopreserved and clonally isolated. They can thus be used to derive a great variety of cell types, including cells of endodermal, mesodermal and ectodermal origin (Shamblott et al., 2001).

The characteristics and the possible use of embryonic stem cells are extensively discussed in this 
issue by Borge and Evers, together with the ethical implications of such area of research. ${ }^{1}$

Adult stem cells have a variable turnover: in the past there was a distinction between permanent proliferative tissues (like blood, intestinal epithelium, skin) sporadically proliferative, and static (like the CNS). However the potential for CNS stem cell proliferation has been unequivocally demonstrated, so that such distinction has become blurred. Furthermore, the question of which signalling pathways are required for stem cell proliferation has been brought to attention and it was interesting to find that the same signals, within individual tissues, may be used both by normal and cancer stem cells, as is the case for Wnt signaling, which comprises a large and highly conserved group of secreted proteins (Nusse, 1999): such discovery has revived the old issue of the relationship between cancer and normal cell proliferation, with a very useful emphasis on the necessity to identify cancer stem cells in order to cure the disease (Reya et al., 2001). A very recent report shows that mutations of a gene which is essential to prevent tumorigenesis in a variety of tissues and organs, the APC (adenomatous poliposis coli) gene, can severely impair the differentiation potential of mouse embryo stem cells (Kielman et al., 2002). Another gene product, a nucleolar protein called 'nucleostemin' as it is acting almost only within the stem cell nucleus, can regulate proliferation of both stem cells and some type of cancer cells (Tsay and McKay, 2002).

In order to understand the variable properties and behaviour of adult stem cells, it is necessary to remember that there is a well-documented genomic diversity of human cells, due to accumulated changes, like chimerism, proviral integration, somatic mutations, epigenetic modifications, etc.: according to Gardner and Lawrence (1986) there are indeed 2 or more genotypes in most adult tissues.

This leads to a well-recognized genomic instability (Pennisi, 2002). As a consequence most tissues are composed of different populations of cells, some still capable of proliferation and self-renewal, others made of post-mitotic cells, only responsible for a specific function (Marshak, Gottlieb and Gardner, 2001). The

\footnotetext{
1 In a very recent report the production of oocytes from mouse embryonic stem cells has been announced (Hubner et al., 2003: Derivation of oocytes from mouse embryonic stem cells, Science, online 10.1126 science 1083452 ).

Although this could be a step towards replacement of reproductive cells by ES cells, more investigations and confirmatory evidence are required.
}

ratio, with aging, is more and more shifted in favour of the former population, in parallel with a progressive fall from the totipotency of zygotic cells to the immutable fate of terminal stages of cell differentiation. Of course this does not apply to germ cells, but oocytes, as before mentioned, are only produced at a very early stage and spermatozoa are produced on demand.

The implications of such findings for the debate on animal clonation and on tissue rigeneration ('therapeutic cloning') are discussed in this issue by Sgaramella and Everts, respectively. Borge and Everts emphasize here the need to regulate the applications of knowledge as its impact on society becomes overwhelming. They also point out the severity of technological problems to be solved before starting large scale clinical trials. It has been found, for instance, that a drawback for the use of ES cells may be represented by their potential (hitherto not admitted) to trigger an immune reaction: there appears therefore no immune-privilege in this respect (Vogel, 2002).

Sgaramella, on the other hand, points out how epigenetics may alter the correspondence between any given DNA portion and eventual genes in different cell lines; this is one of the reasons why animal cloning, occasionally possible, is invariably inefficient.

At this point, and while more experimental evidence is required, we may consider the changes in the proliferative potential and differentiation capacity of somatic stem cells, which have been observed in recent years.

Great plasticity has been indeed reported for stem cells, which were believed to be committed to a particular lineage, like haemopoietic stem cells and neural stem cells, suggesting a so-called phenomenon of 'transdifferentiation'. In order to explain such events, it is thought that the tissue micro-environment may play an important role, as signals from adhesion proteins have been demonstrated (Watt and Hogan, 2000). However, in some instances, doubts have been raised about the reality of such transformation: cell fusion has been implicated, as shown by Terada in this issue. More doubts on transdifferentiation are raised from other quarters (Pearson, 2002).

In this respect neural stem cells (NSC) have been the subject of special investigation in the recent years. They have been indeed successfully isolated from both adult animal and human brains by selective growth as floating clusters of neurospheres in culture (Figure 1), where they can be easily amplified, differentiated (Figure 2) and cryopreserved for future use (Caldwell et al., 2001). Characteristics and plasti- 


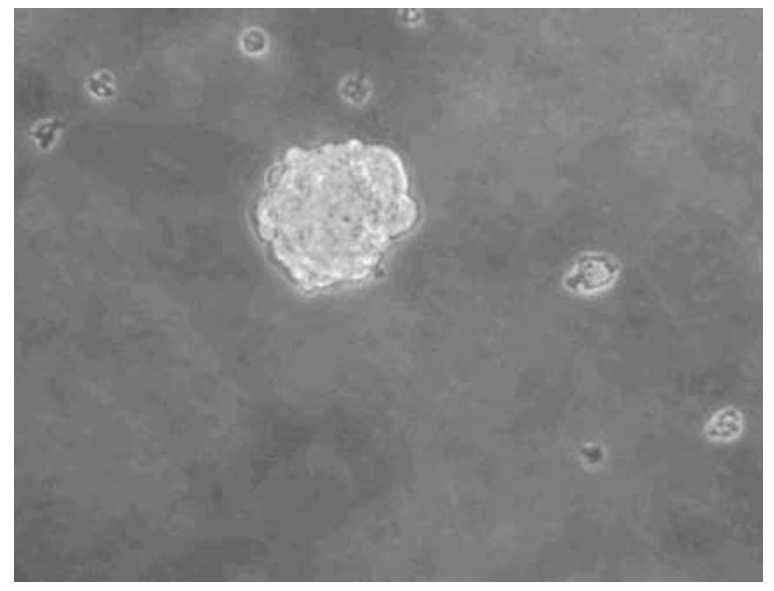

Figure 1. Neural stem cells proliferate in suspension as a clonal cluster of cells called neurosphere, which is heterogeneous and composed by a mixed populations of progenitors and stem cells. In the presence of mitotic growth factors (EGF, $20 \mathrm{ng} / \mathrm{ml}$ ) the stem cell compartment is amplified by symmetric divisions.

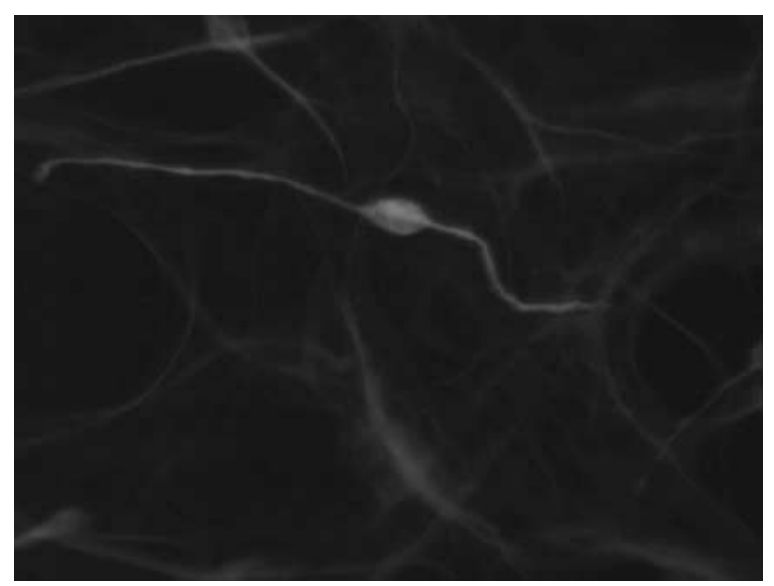

Figure 2. After adhesion to a substrate, such as laminin or polyornithin, neural stem cells can differentiate in the presence of serum $(1 \%)$ and after withdrawal of the growth factor. This is a staining for $\beta$-tubulin III, a specific protein expressed by neurons. Several papers have reported that stem cell-derived neurons, differentiated even at late passages in vitro, possess all the typical electrophysiological characteristics of primary cultures neurons.

city of NSCs 'in vitro' have been described in details (Temple, 2001) and it has been proved that the adult nervous system can generate 'in vivo' progenitors and new neurons at least in two regions: the dentate gyrus and the subventricular zone. More recent data indicate that adult multipotent progenitors could exist quiescently in other mammalian brain regions, including the frontal cortex of non-human primates (Ourednik et al., 2001).
Neurogenesis is regulated by environmental stimuli and can be responsive to brain injury, therefore neural stem cell persistence and plasticity may indicate a possible endogenous repair program which could be damaged in neurodegenerative diseases. So CNS stem cells have potential therapeutic applications as an autologous source of tissue engraftment (stem cell transplantation) and/or self-repair by sistemic administration of neurogenic and neuron growth-promoting factors (such as bFGF and neuroimmunophilin ligand) able to stimulate normally quiescent stem/progenitor populations in order to replace specific populations lost in neurodegenerative diseases.

Perspectives and problems related to a clinical use of neural stem cells have been extensively described (Rossi and Cattaneo, 2002) and NSC have been successfully injected in different animal models of diseases where they have reconstituted missing functions (such as myelination in shiverer mouse) (Yardava et al., 1999). In addition, stem cells can be genetically manipulated and transplanted giving rise to a modified differentiated progeny able to express specific genes (Martinez-Serrano and Biorklund, 1997). NSC migration capability has been exploited for the treatment of brain tumors, for re-establishment of lost connections in injured spinal cord (Wu et al., 2002) and for prospective treatment of some brain tumors, as described in this issue by Prof. Finocchiaro's group: main focus from these Authors is on the potential for stem cell applications in glioblastoma multiforme, a very aggressive neoplasm, and leukodistrophy, a metabolic disease of the white matter. It is interesting that in this context not only neural but also haemopoietic stem cells may be used.

Haemopoietic stem cells have been widely used in the past to reconstitute haemopoiesis in patients receiving lethal radiotherapy, therefore showing a complete rigenerative potential; among the sources used were bone marrow (BM), peripheral blood and cord blood (Thomas, 1991; Winter, 1997).

How frequent are such progenitors in blood or blood-forming cells? The exact number is still uncertain, but a comparison between the frequency of early clonogenic cells from different sources (B.M., peripheral blood and cord blood) has shown a significantly higher amount of such precursors in cord blood samples, while the peripheral blood gives the smallest yield (Eridani et al., 1998).

Cord blood clonogenic cells have been used for some time to treat patients with haemopoietic ailments, including genetic disorders and leukemia 
(Gluckman et al., 1997); it has actually been shown that cycling fetal and neonatal stem cells have the same functional capability of quiescent cells from adult sources (Wilpshaar et al., 2000) A note of caution for cord blood cells has been however sounded by Mel Greaves and his group. They actually show that chromosome translocations characteristic of pediatric leukemia often arise before birth (while additional events are required post-natally for the leukemic development): leukemia fusion genes are actually present in cord blood at a frequency much greater than the risk of the corresponding leukemia (Mori et al., 2002). Such finding may raise some concern about using stored cord blood for transplantation purposes.

A special type of bone marrow derived cells are mesenchymal stem cells, which have been shown to be multipotent progenitors, capable of large expansion in vitro and also able to differentiate, when injected into a blastocyst, into a variety of somatic cell types (Jiang et al., 2002).

The relative merits of haemopoietic stem cells are dealt by Clark et al. (for the bone marrow) and De Wynter (for cord blood).

A series of investigations have also addressed stem cells which may have important applications in clinical trials for tissue rigeneneration; among areas covered in this volume are the the hepatocyte precursors and pancreatic islet stem cells. Neil Theise underlines in this volume the distinction between the intra-hepatic and extra-hepatic stem cell compartments, as the latter may include populations of cells from the bone marrow: the balance between such compartments is determined by the nature and the extent of insults of different kinds.

Pancreatic regeneration was the subject of early research in the Seventies by William Chick in Boston, who tried to obtain a new source of insulin production by inserting islet cells into diabetic patients: there are now promising experiments to reach the same goal by using islet cell precursors rather then mature cells, as described here by Prof. Soria and his group.

Muscle stem cells have also received a lot of attention in recent years, both for their possible use in peripheral muscular disease and as cardiomyocite precursors, which may be be used for relief of ischemic heart disease (Ferrari et al., 1998). Some progress has been made in these areas, as discussed in this issue by White and Grounds, from Australia, but there are still severe limitations before stem cells can be seriously considered for clinical use in human myopathies.The most promising approach seems at the moment the possible activation of 'resident' stem cell subpopulation within skeletal muscle.

Dr. Fukuda, from Japan, describes cardiomyocite precursors, belonging to a cell line isolated from murine bone marrow stromal cells; they show a typical cardiomyocyte ultrastructure and spontaneous beating, which is either increased or blocked by treatment with different compounds.

Chondrocyte precursors are also being investigated for possible use in providing efficient cartilage where it is lacking or for repairing damage: experimental research in this area is described in this issue by Rohwedel and coworkers, who obtained pure chondrocyte cultures from cellular aggregates known as embryoid bodies; from these cultures it was possible also to obtain other mesenchimal cell types.

Epithelial stem cells, particularly skin cells, have been pinpointed as one of the simplest sources of stem cells: moreover they may be greatly amplified in culture and used not only for repairing epidermal lesions, but also reprogrammed to form other somatic cell types (Fuchs and Segre, 2000). A special type of epithelial cells, namely prostatic stem cells, are the subject of a review in this issue by David Hudson.

Transplantation of stem cells is also now experimented in a variety of different conditions: one interesting area is represented by congenital immunedeficiency disorders, where interesting results have been obtained.

Another very interesting area of research is stem cell transplant attempt as a form of cancer immunotherapy (Appelbaum, 2001): encouraging results are presented by Richard Childs of NIH in a series of solid tumours in advanced stage.

Altogether it appears that stem cell research is in full bloom, accompanied by a flurry of contrasting views on both aims and means (Weissman, 2002): it is significant, for instance, that in the U.S. a fierce debate has occurred at the Meeting of the President's Council for Bioethics: a temporary (4 yr) moratorium on cloning for therapeutic research was discussed and finally approved with a slim majority, of 10 to 7 . It is also worth noting that for some members of the panel such moratorium only means a pause to allow for regulations to be established while for others it may allow to conduct a campaign for a permanent ban (Hall, 2002)

The issues at stake are thus very complex and it is easy to predict that debates and discussions will go on for a long time to come: however stem cell research is bound to continue, supplying a wealth of information 
which will be of value in deciding the future course of action.

\section{References}

Appelbaum FR (2001) Haematopoietic cell transplantation as immunotherapy. Nature 411: 385-389.

Blau HM, Brazelton TR \& Weismann JM et al. (2001) The evolving concept of a stem cell: Entity or function? Cell 105: 829-841.

Caldwell MA, He X \& Wilkie N et al. (2001) Growth factors regulate the survival and fate of cells derived from human neurospheres. Nature Biotech 1: 475-479.

Eridani S, Mazza U \& Massaro P et al. (1998) Cytokine effect on ex vivo expansion of haemopoietic stem cells from different human sources. Biotherapy 11: 291-296.

Eridani S (2001) Versatile stem cells, young and old. A review. Cytotechnology 35: 137-143

Ferrari G, Cusella Deangelis G \& Coletta M et al. (1998) Muscle regeneration by bone marrow-derived myogenic progenitors. Science 179: 1528-1530.

Fuchs E \& Segre EA (2000) Stem cells: A new lease of life. Cell 100: $143-155$.

Gardner RL \& Lawrence PA (1986) Single cell marking and cell lineage in animal development. Philos Trans Royal Soc London 313: 1-187.

Gluckman E, Rocha V \& Boyer A et al. (1997) Outcome of cord blood transplantation from related and unrelated donors. N Engl J Med 337: 373-381.

Hall SS (2002) President's bioethics council delivers. Science 297: 322-324.

Ivanova BN, Dimos JT \& Schaniel C. et al. (2002) A stem cell molecular signature. Science 298: 601-607.

Jiang Y, Jahagirdar BN \& Reinhardt RL et al. (2002) Pluripotency of mesenchymal stem cells derived from adult marrow. Nature (advance on-line publication, 20-6-2002).

Kielman MF, Rindpaa M \& Gaspar C et al. (2002) APC modulates embryonic stem cell differentiation by controlling the dosage of beta-catenin signaling. Nat Genetics 32: 594-605.

Lajtha L (1979) Stem cell concepts. Differentiation 14: 23-34.

Lovell-Badge R (2001) The future for stem cell research. Nature 414: 88-91.

Marshak DR, Gottlieb D \& Gardner RL (2001) In: Stem Cell Biology, Cold Spring Harbor Laboratory Press, New York, pp. 1-16.

Martinez-Serrano A \& Björklund A (1997) Immortalized neural progenitor cells for CNS gene transfer and repair. Trends Neurosci 20: 530-538.

Mori H, Colman SM \& Xiao Z et al. (2002) Chromosome translocations and covert leukemic clones are generated during normal fetal development. Proc Natl Acad Sci USA 99: 8242-8247.

Nusse R (1999) WNT targets: Repression and activation. Trends in Genetics 15: 1-3.
Ourednick V, Ourednick J \& Flax JD et al. (2001) Segregation of human neural stem cells in the developing primate forebrain. Science 293: 1820-1824.

Pennisi E (2002) Charting a genome's hills and valleys. Science 296: 1601-1602.

Ramalho Santos M, Yoon S \& Matzusaki Y et al. (2002) Stemness: Transcriptional profiling of embryonic and adult stem cells. Science 298: 597-600.

Reya T, Morrison SJ, Clarke MF \& Weissman IL (2002) Stem cells, cancer and cancer stem cells. Nature 414: 105-111.

Rossi F \& Cattaneo E (2002) Neural stem cell therapy for neurological diseases: Dreams and reality. Nature Reviews 3: 401-409.

Shamblott MJ, Axelman J \& Littlefield JW et al. (2001) Human embryonic germ cell derivatives express a wide range of developmentally distinct markers and proliferate extensively in vitro. Proc Nat Acad Sciences USA 98, 113-118.

Smith AG (2001) Embryonic stem cells in Stem cell biology. Edited by Marshak DR, Gardner RL \& Gottlieb D, New York: Cold Spring Harbor Laboratory Press, 205-230.

Snyder EY \& Vescovi A (2000) The possibilities/perplexities of stem cells. Nature Biotech 18: 827-828.

Temple S (2001) The development of neural stem cells. Nature 414: 112-117.

Tsai R \& McKay R (2002) A nucleolar mechanism controlling cell proliferation in stem cells and cancer cells. Genes Develop 16: 2991-3003.

Thomas ED (1991) Frontiers in bone marrow transplantation. Blood Cells 17: 259-267.

Van der Kooy D \& Weiss S (2000) Why stem cells? Science 287: 1439-1441.

Vogel G (2002) Stem cells not so stealthy after all. Science 297: 175-176.

Yardava BD, Billinghurst LL \& Snyder EY (1999) Global cell replacement is feasible via neural stem cell transplantation: evidence from the dysmyelinated shiverer mouse brain. Proc Natl Acad Sci USA 96: 7029-7034.

Watt FW \& Hogan BLM (2000) Out of Eden: Stem cells and their niches. Science 287: 1427-1430.

Wilpshaar J, Falkenburgh JHF \& Tong X et al. (2000) Similar repopulating ability of mitotically active and resting umbilical cord blood CD34+ cells in NOD/SCID mice. Blood 96: 2100-2107.

Weissman IL (2000) Stem cells: units of development, units of regeneration, and units of evolution. Cell 100: 157-168.

Weissman IL (2002) Stem cells-scientific, medical and political issues. New Engl J Med 346: 1567-1579.

Wilson EB (1898) The cell in development and inheritance. MacMillan, New York City.

Winter JN (ed.) (1997) Blood stem cell transplantation. Kluwer Academic, Boston.

Wu S, Suzuki Y \& Kitada M et al. (2002) New method for transplantation of neurospheres cells into injured spinal cord through cerebrospinal fluid in rat. Neurosci Lett 318: 81-84. 